Christopher Fox

\title{
Some recent British string quartets
}

\begin{abstract}
Eight recent works for string quartet are considered with particular emphasis on the innovative characteristics of each work and the relationship between the works and the ensembles for which they were written.
\end{abstract}

\section{KEYWORDS}

String quartet; Arditti Quartet; Kreutzer Quartet; Smith Quartet; Bozzini Quartet; Jonathan Harvey; James Clarke; Howard Skempton; Richard Glover

The string quartet holds a unique place in western European art music; no other flourishing instrumental ensemble has such a long, continuous history. It is a history which reaches back into the middle of the $18^{\text {th }}$ century but it is also a history with ambiguity at its heart, because the string quartet is both a medium and a form. The medium is fascinating-intimate, homogenous, rich in tonal possibilities, but unbalanced in favour of treble instruments-and the form is represented by the extraordinary compendium of masterpieces which we now regard as the ensemble's core repertoire, from Haydn to Mozart to Beethoven, Brahms, Bartok and Shostakovich. I want to examine the creative responses made by a number of British composers to both the medium and form of the string quartet, focusing exclusively on works created since 2000.

In Britain in the period after the end of the Second World War the history of the string quartet placed it at the centre of classical music culture. The arrival of emigré musicians such as the members of the Amadeus Quartet from continental Europe, the creation of the BBC's Third Programme as a radio station devoted to classical music, the institution of state-support for professional concert-giving through the newly-founded Arts Council, and the growth in the number of universities, all contributed to a burgeoning of opportunity for serious music, and no form is more serious than the string quartet. The conservatoires trained string players to form quartets which could then be sustained by a touring circuit of music clubs around the country, by broadcasting, and by institutional residencies. In the early 1960s, as a symbol of its aspiration to be a model of modern civic development, Harlow New Town adopted the Alberni Quartet, perhaps the finest British quartet to emerge in the 1960s, and by the 1970s any self-respecting British university music department had a string quartet in residence: the 
Fitzwilliams at York, the Chilingirians at Liverpool, the Lindsays at Sheffield and then Manchester, and many others.

The cynic might observe that these residencies also had some very obvious advantages for the institutions: string quartets added only four salaries to the payroll and in academia these might well buy some instrumental teaching and the occasional stiffening of the university orchestra; above all, the diversity and academic probity of the quartet repertoire could supply the backbone for the university concert season. This intimate connection between academia and string quartets sometimes embraced new composition too, at least in those departments which included what Kingsley Amis describes in his great university satire, Lucky Jim, as a 'local composer' (Amis, 53). At York David Blake wrote his String Quartet No.2 (1973) for the Fitzwilliam Quartet and during my time as an undergraduate at Liverpool the Chilingirian Quartet played Hugh Wood's String Quartet No.2 (1970) and encouraged him to write his String Quartet No.3 (1978).

But Blake and Wood, both significant figures in the British music scene of that era, were unusual. Relatively few British universities in the 1960s and '70s employed composers and, if they did, it was not to teach or practise their art; composition was at the margin of the academic curriculum. The commissioning of new music was similarly at the margin of most string quartets' activity. Audiences wanted - as far as anyone knew-to hear great works from the core repertoire and when new works were introduced the expectation was that they should complement this repertoire, the context within which they would be heard. The results were generally mediocre, composers grappling with form and history to produce what Lucky Jim so wickedly dismisses as 'scurrying tunelessness' (Amis, 53).

All this was to change. The advent of the new music string quartet, led in the UK by the Arditti Quartet, broke the link between the Classical and Romantic repertoire and the composition of new works for the medium. Instead of composers being commissioned to write the lone new work in a concert programme of acknowledged masterpieces for an ensemble whose players, although highly-skilled, might well complain if they were asked to change 
metre, play col legno, find remote natural harmonics, or re-tune too often, they could write for musicians who would happily deal with all these challenges. Instead of writing music which attempted to inhabit familiar forms, composers were invited to re-imagine what the string quartet as an ensemble might play or, at least, to continue a process of innovation which might be traced from Webern's Five Pieces, op.5 to Xenakis's ST/4.

In the wake of the Ardittis (and in the USA their near-contemporaries, the Kronos Quartet) came other quartets with a similar focus on new work and it is with music written for these new music string quartets that this article is concerned. Mainstream repertoire quartets in the UK continue to commission new works and some of these have met with considerable success, perhaps none more so than Thomas Adès's seven-movement divertimento, Arcadiana (1994), commissioned by the Endellion Quartet, but I would argue that it is in the works written for specialist new music ensembles that the most significant new music for string quartet can be found.

As well as giving an account of a number of these new works I also want to consider the relationship between different ensembles and the music created for them by British composers, so the works I have chosen to discuss were composed for four different quartets, the Arditti, Smith, Kreutzer and Bozzini Quartets. The Ardittis are represented by Jonathan Harvey and James Clarke, the Kreutzers by Michael Finnissy, the Smiths by Howard Skempton and Christopher Fox, and the Bozzinis by Richard Glover. When Richard Toop invited me to write this article I also thought that, as someone who writes music as well as words about music, it would be interesting not just to write about the string quartet but at the same time to write a string quartet which might, in turn, be music about the string quartet. I had already worked with the Arditti, Kreutzer and Smith Quartets; fortunately the Bozzini Quartet agreed to commission a new work from me and so that provides a second example for them.

Irvine Arditti triggered the renewed interest in the string quartet as a medium for new music, not only by assembling a quartet of musicians with the technical skills to tackle existing works by Carter, Scelsi and Xenakis, but also 
by commissioning new works. The Arditti-commissioned quartets of Ferneyhough and Dillon are discussed elsewhere in this issue of Contemporary Music Review but it is appropriate that the first work discussed in this article should be by Jonathan Harvey (1939-2012), the first composer to write for the Ardittis. Harvey's Quartet No.1 (1977) was also something of a breakthrough for its composer since it was the first work in which his mature compositional language emerged, fusing elements of serial organisation with overtone-based harmonic and melodic construction.

Quartets Nos.2 and 3 followed, in 1988 and 1995 respectively, and are characterised by an improvisatory fluidity both in form and instrumental writing; a fine cellist himself, Harvey's writing for strings was always particularly inventive. But String Quartet No.4 (2003) is perhaps his most significant work in the medium, not only because of its length-it runs for more than 35 minutes-but also because of its combination of live instrumental playing with real-time signal processing. Throughout his career Harvey was fascinated with the potential of electronic musical resources to enable instruments-and, by extension, listeners-to transcend their physical, corporeal existence, to be shifted in shape and space, and in String Quartet No.4 live digital processing moulds new sonic entities out of fragments of the instrumental sound. More importantly for the imagery of the work, the computer software mobilises these new sounds, letting them fly around the auditorium as if carried by the wind.

Formally, the work is quite straightforward: a succession of five extended episodes or, the composer's term, 'cycles', each of which emerges out of different forms of white noise-bowing on the tail-piece, at the bridge, on the body of the instrument-and the music gradually develops, as if through a process of accretion, accumulating pitches and gestural material to a series of climaxes. As the piece unfolds the electronic transformations become more apparent and more elaborate-structural entities in their own right rather just commentaries on the instrumental action-until at the end the music returns once more from pitch to noise. The electronic sounds rotate at what Harvey descibes as 'stroboscopic speeds [...] mostly around twenty-three times a second'; but, as he also points out, this fast rotation produces 'an illusion [...] 
of slowly turning' (Harvey, 2012, 52), like images of turning wheels in early motion pictures. It's a powerful image which, not for the first time in Harvey's work, recalls the closing minutes of Stockhausen's Kontakte, but Harvey's special achievement in String Quartet No.4 is to make such an intimate connection between the instrumental and electronic soundworlds.

As Europe's leading new music quartet for at least three decades, and as a quartet whose aesthetic has always been tilted towards the progressive version of modernism favoured by grand institutions of European culture such as IRCAM, it is perhaps surprising that the Arditti repertoire does not include more works like Harvey's String Quartet No.4 which combine live performance with electronics. On the other hand, one of the attractions of the string quartet for promoters is that it is usually a medium with no add-on costs-no extra players, no extra instrument hires—so why complicate matters with microphones, cables, loudspeakers and technical staff? Whether through principle or pragmatism the bulk of the Arditti repertoire is made up of works for four unamplified instruments and James Clarke (b.1957) is one of the most strikingly original British exponents of this unadulterated version of the medium.

Clarke's two string quartets have been written for the Arditti Quartet and both were co-commissioned by the Huddersfield Contemporary Music Festival, the String Quartet (2003) with Ars Musica (Brussels) and String Quartet No.2 (2009) with Maerzmusik (Berlin). A cursory glance at the score of either work might suggest that these are typical examples of the EuropeanNew-Music-Festival genre-hyper-active instrumental textures, eighth-tone accidentals and irrational rhythms blurring pitch and rhythmic conjunctions-but in both works the ear tells a different story.

At just ten minutes long, the String Quartet has a concentrated intensity typical of much of Clarke's music. It opens with a solo for the first violin over an open D string drone in the viola, the violin making a series of gestures-a repeated double stop, then quick glissandi-which sketch round the outline of the $3 / 4$ metre and a $D$ triad. The tempo, sustained throughout the piece (apart from a brief rallentando and accelerando just before the coda), is quick 
(120bpm) and the pulse soon becomes more discernible when, four measures into the piece, the other instruments join the first violin, with one instrument always articulating the quarter-note beat. But what gives the music its propulsive energy is Clarke's constant warping of the triple metre: for four measures from measure 8 (and a distinct four-measure phrase pattern underpins the structure of the entire quartet) all four instruments play 13:12 groupings within the 3 / 4 measure, a dotted eighth-note followed by five regular eighth-notes, giving the effect of an exaggerated emphasis on the downbeat.

Soon the effect is reversed. 5:6 groupings-dotted quarter-note followed by quarter-note-are introduced by the violins at measure 17 and by measure 25 have spread to all four instruments, giving the effect of an extended up-beat. Later still the metre is subtly varied-13/16 at measure $106,7 / 8$ at measure 113,2 / 4 at measure 116-but the underlying sense of a triple metre is always preserved, particularly through the use of repeated figuration. Example 1 shows the cello part from measures 109 to 114; each measure begins with a double-stop, whose lower note is the lowest note within the measure; the upper note is an E for four measures and then, after the change in metre, a D.

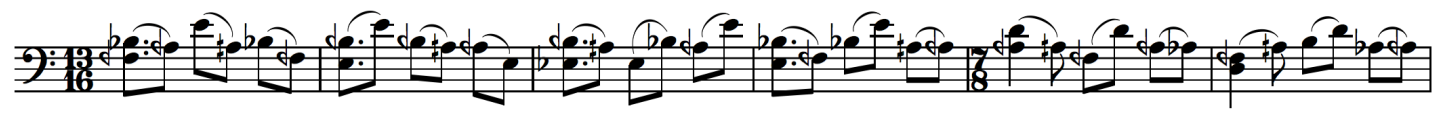

Example 1, James Clarke, String Quartet (2003); cello part, measures 109-114

Similar strategies are used throughout the piece and in every case echo musical devices familiar from folk music: repeated figures in 'melody', 'bass' and 'rhythm' parts, pitch shifts at structural points (the E to D in the cello example above, for example), dramatic changes in texture (flautando dominates from measure 176 to 196 , to be succeeded by aggressively repeated double-stops for the next 36 measures). Yet at no point does Clarke reference a specific folk-culture; instead he creates a work in which the forward impetus of dance music is distilled and maintained through a dizzying series of variations. 
Michael Finnissy's String Quartet (1984) also offers a distillation of aspects of folk music and, like Clarke's String Quartet, it was commissioned and premiered by the Arditti Quartet; but since they gave the work its first run of performances it has been absent from their repertoire. It was subsequently taken up by the Kreutzer Quartet, a younger British quartet based around the first violinist, Peter Sheppard Skærved, and in 1998 the Kreutzer Quartet released a CD (Metier MSV92011) of all Finnissy's current music for string quartet: Nobody's Jig (1981), the String Quartet, Plain Harmony (1993), Sehnsucht and Multiple forms of constraint (both 1997).

The CD's title—not 'String Quartets' but 'Works for string quartet'—was significant because, although each work uses the four instruments of the ensemble, none of could be said to be a 'String Quartet' in any conventional sense. Nobody's Jig is a bravura display of the heterophonic writing at which Finnissy has always excelled, the four instruments each playing from an individual part with no regard for what the others are doing, Plain Harmony was originally written for the amateur music organisation COMA and can be played by a variety of different instrumental ensembles, Sehnsucht is a beautiful little meditation on a Brahms song, and Multiple forms of constraint sets a soloist (the first violin) against a trio (the rest of the group). Even the work entitled 'String Quartet' eschews familiar conceptions about the medium and form of the quartet: it is an extended single movement made up of a series of sharply differentiated sections, in each of which the four instruments generally function individually rather than in consort.

Nearly ten years later, in response to a Kreutzer commission, Finnissy returned to the string quartet, eventually producing not one but two works, and the Second and Third String Quartets engage with the string quartet form and its history much more directly, albeit in strange and radical ways. There is another difference between these two works and Finnissy's earlier quartet output; whereas the works collected on the Kreutzer's 1998 CD rather neatly capture the changes in Finnissy's compositional preoccupations over a period of nearly 20 years, the Second and Third String Quartets have a much more convoluted and interconnected genesis and, although very different in scale, share the same concerns. According to Finnissy the conception of the Third 
Quartet, written between 2007 and 2009, actually preceded that of the Second Quartet (2006-7): 'I had intended to write the 3rd quartet a little earlier than I did - and, because of time constraints and knowing that it was going to be a much more extended piece, I wrote the 2nd quartet instead.' (Finnissy, 2010)

The question of scale is important. Many Finnissy works derive both material and structural principles from older music by other composers and in these two quartets Finnissy turned to Haydn and Bruckner respectively. But when time constraints deflected him from the extended work he had been planning Finnissy decided to save Bruckner for later and instead base the Second String Quartet 'on a compact Haydn model'. Consequently the Second String Quartet has a more straightforward relationship to the string quartet tradition than anything else in Finnissy's output; not only is the scale of its formal ambition based on Haydn but Finnissy also makes extensive use of references to Haydn's op.64 No.5. Discerning listeners will hear Haydn-like melodic and rhythmic contours emerge at various points and at around 5'21" in the Kreutzer's CD recording of the work (NMC D180), for example, a distorted version of the Finale of Haydn's quartet comes into focus, its allegretto tripletime rhythm preserved, complete with appoggiatura-decorated upbeat, but its melodic shape transformed. The effect is typical of later-Finnissy, as if we are listening to the aural equivalent of a series of photographic multiple exposures, some exposures more in focus than others, not all of them quite fitting the frame.

Part of the reason for this blurring is that the Second String Quartet has no score, just a series of parts, each prefaced by the instruction that 'it is intended that the parts should drift slightly apart, and definitely not seem calculatedly or rigidly aligned'. This aspect of the music links the Second String Quartet with the heterophony of Nobody's Jig, Finnissy's earliest work for the medium, but it also connects the Second Quartet with much earlier examples of polyphonic ensemble music from the days when individual part-books had not yet been supplanted by a unifying score. One of the delights of the Second Quartet is this dialogue between the quartet tradition and even older forms of consort music, the ascendancy of the quartet tradition asserting itself at each of the 'gathering moments' which regularly pull the music back together. 
If the Second String Quartet has the dimensions of a classical quartet then the Third String Quartet can be heard as stretching out to emulate the quartet's larger, symphonic brother, with a form modelled not on Haydn but on Bruckner. Finnissy says that he has 'loved Bruckner's music since the first time I heard his Ninth Symphony at the Proms (probably about 1963). I just love the harmony and how he builds up structural shapes' and in the opening of the Third String Quartet the Brucknerian model is very clear. Finnissy planned the work's structure as a series of blocks, proportionally organised according to a numerical cipher based on the name of the work's dedicatee: A.M.A.N.D.A.B.A.Y.L.E.Y. The recurrence of the letter A also suggested a way of interrelating the structural blocks, so that all the A sections are variants of the opening. This grand opening always combines both atonal and tonal material and the tonal material—what Finnissy describes as 'the grandly tragic bits with key-signatures' —always alludes to Bruckner's First and Second Symphonies. In typical Finnissy style, however, the transcription of these 'bits' has seriously complicated them: the Third String Quartet begins with a dense adagio juxtaposing many different melodic lines, each following its own chromatically-inflected path through a different key.

But Bruckner symphonies were not Finnissy's only model. He also wanted to reference Schoenberg's String Quartet No.2 and its introduction of a fifth member within the ensemble. Schoenberg adds a singer who announces that 'Ich fuhle Luft von anderen Planeten' and Finnissy too wanted to 'get involved in exploring the idea of the "air from another planet", and what this might, or could mean'. He says that, in 'trying for an unforced sort of equivalent, and-trying to keep it more low-key-of somehow embracing 'natural' elements (OUR planet, but the OTHER so-called musical voices of our planet)', he found himself looking for something which 'would contrast with the abstract design', the proportional structural scheme he had imposed on the music.

Eventually Finnissy realised that the 'natural element' should be birdsong; he started transcribing it and it became for him 'a reflection on the vocal conclusion to Schoenberg's Second Quartet'; as he rather wrily observed, 'had 
it been my second quartet too, this might have been clearer!' Just as Schoenberg reserves the first vocal entry until the third movement of his quartet, so too Finnissy holds back the first appearance of his additional voices until the latter stages of the Third String Quartet. There, however, the similarity ends. The voice in Schoenberg's quartet may extend the ensemble but it does so within the aesthetic space of concert music; Finnissy extends his quartet not just with transcriptions of birdsong but with recordings of birdsong too-not just 'other' voices but voices which take us into an 'other' space beyond the concert hall, beyond music itself.

The effect is extraordinary: at first the recordings seem like an intrusion, interrupting the flow of Finnissy's invention, but as the piece moves towards its conclusion it is the instruments of the quartet which come to seem intrusive, even though they are playing music based on transcriptions of birdsong. Finnissy's birdsong transcription method is simple: 'just pencil and paper + ears' $[. .$.$] the recordings are from my garden, and the nearby Downs,$ very early in the morning.' But the passage from bird to ear to paper to instrument, however objectively done, involves human agency and the juxtaposition of transcriptions and field recordings makes clear that agency. As Finnissy's Third String Quartet plays itself out-there is no real conclusion-we are left to muse on humanity's capacity for elaborate artifice and to compare it with the unknowable, yet evidently essential purpose of this other 'song'. There is something valedictory about the end of the Third String Quartet and one wonders at the significance of the work's trajectory, from its grandly symphonic opening to this final, strange, private dialogue between composer and birds.

The Kreutzer Quartet initially took over Finnissy from the Arditti Quartet but it is hard to imagine the Ardittis adding Finnissy's Second and Third String Quartets to their repertoire. Finnissy's synoptic vision of musical history, in which music from all eras and all cultures may at any moment become a point of reference, is at odds with the Ardittis' more conventionally modernist view of musical history as a progressive narrative. It is one of the interesting features of contemporary string quartet music that, while the Arditti and Kronos Quartet may have established the concept of a string quartet devoted 
to new music, few of the new works written for them, or for any of the younger ensembles formed in their wake, have become a shared new music quartet repertoire.

In an era of brand-awareness, each ensemble uses their choice of composers and works as a mean of defining themselves in the market-place. It would be an interesting exercise, but beyond the scope of this article, to observe this process in action through a detailed mapping of composers, works, quartets and performance venues. It is obvious, however, that the Kreutzer, Diotima and JACK Quartets have each adopted some elements of the Arditti repertoire and are very evidently in competition with one another for the same European new music festival bookings. Similarly the Smith Quartet began life as a British version of Kronos, mixing local minimalists like Steve Martland and Graham Fitkin in with Reich, Glass and Feldman works originally created for the Kronos.

In 2005 the Smith Quartet and Roger Heaton gave a performance of my clarinet quintet (1992) at the Huddersfield Contemporary Music Festival. It was an enjoyable experience and so the Festival and the Smiths commissioned me to write a new quartet for the following year's festival. I wrote 1-2-3, a work which explicitly takes off from the Smiths' minimalist connections by recalling Terry Riley's In C (albeit a tone higher) in its very first measures (see example 2). As the music evolves it becomes clear that there are two significant differences between Riley's work and mine: In $C$ is modal, each repeated phrase based on the same blue-note-inflected $C$ major scale, whereas 1-2-3 is based on a series of interlocking intervallic relationships; In $C$ is a work which can be played by any collection of instruments whereas the formal processes of 1-2-3 are locked into the acoustic characteristics of the instruments of the string quartet. 


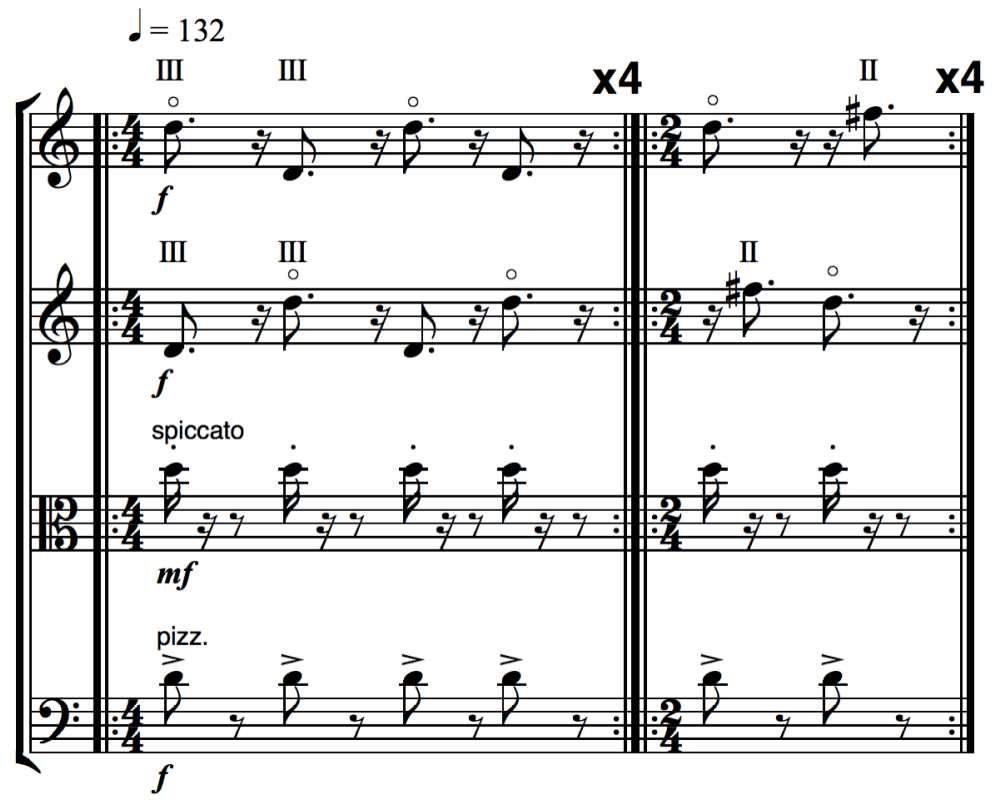

Example 2, Christopher Fox, 1-2-3, measures 1-2

As someone whose early experience of music was shaped by attempts to get the complicated acoustics of the french horn to behave, I have always been fascinated by the tension between the physical characteristics of musical instruments and the music we ask them to play. The relatively straightforward act of bowing a string can yield such complex sounds, simply by varying the speed, position and weight of the bowing, yet most composed string music within the European art music tradition chooses to suppress these complexities by stopping the string with the left-hand and using a uniform bowing action. Even today, composers who include the higher natural harmonics in their string-writing are likely to be told by conventionally-trained players that the same notes can be achieved 'much more securely' using artificial harmonics.

This tension, between the 'natural' phenomenon that is a bowed open string and the 'artifice' of stopped strings and scales, plays a key role within the structure of 1-2-3. As example 2 demonstrates, open strings and their harmonics are an important part of the work's soundworld from the beginning and, as the music progresses, the sounding of open strings regularly brings the music to a temporary halt, from which it usually recovers with a new version of the opening figure. Eventually, however, these attempts to revive the flow of the music become less successful and after 
about eight minutes it tips over into a quite different soundworld, a world of varied bowing directions and pressures, quasi-random melodies on the high harmonics, tappings and knockings. There can be no way back, although, as the title 1-2-3 implies, the work has a final coda which attempts some sort of final synthesis-the pitch sequence unfolded in the first section re-presented as a series of solo melodies over sustained drones.

Although it was perhaps the Romanian musicans of the ConTempo Quartet who got closest to the inchoate musical language of the middle section when they played the work in 2007, the Smith Quartet gave a series of fine premiere performances of 1-2-3 in which the spirits of Riley and Glass were very audibly present. But a work which deliberately opposes such different musical territories—to put it at its crudest, 'systems minimal', 'spectral', 'holy minimal' - is likely to challenge many ensembles and the Smith Quartet were evidently more comfortable in Howard Skempton's Tendrils (2004), a cocommission from the BBC and the Huddersfield Contemporary Music Festival which they premiered in Huddersfield in November 2004.

In a 2012 Tempo interview with John Fallas, Skempton (b.1947) says of Tendrils that it is 'in a way the piece of which I am proudest, because I went out on a limb and created a 20-minute movement which is completely seamless.' (Fallas, 27) For Skempton, a composer who made his reputation as a miniaturist, the scale of Tendrils may initially appear unusual but closer examination reveals that the work is made up of a series of short sections, 55 in all, whose beginnings and endings always overlap. Continuity is assured by the consistent use of the same pitch material-the third of Messiaen's modes of limited transposition (see example 3) —and the same compositional process-each new section involves a series of canonic entries at the minor sixth, with freely-composed harmonisations added so that, as Skempton puts it, the 'joins are disguised'. The canons are always begun by either the cello or the first violin, each cello entry starting low and generally moving higher, the alternating first violin entries answering with a general movement downwards. 


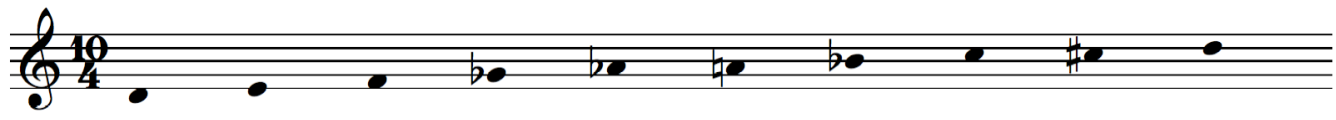

Example 3, Third mode of limited transposition

In a fascinating exchange of emails between Skempton and the composer Bryn Harrison, Skempton explains that his method was to compose 'each canon as a single bar. The top four staves of the first canon are what you see at bar 4. [...] In bar 5, the original entry disappears and the cello has a new line [see example 4]. The joins are disguised by extra intuitively-composed material (the violin 2 line in bar 6, for example). The initial melody of each canon was composed intuitively, in the light of the previous one. With each new canon, there was the possibility of a change of transposition, or (on occasion) time signature.' Skempton adds that 'the effect of transposing each entry up (or down) a minor sixth' is 'miraculous' and that 'the result is kaleidoscopic, giving the impression of continuous modulation'. (SkemptonHarrison, 5 April 2011, 00:46).

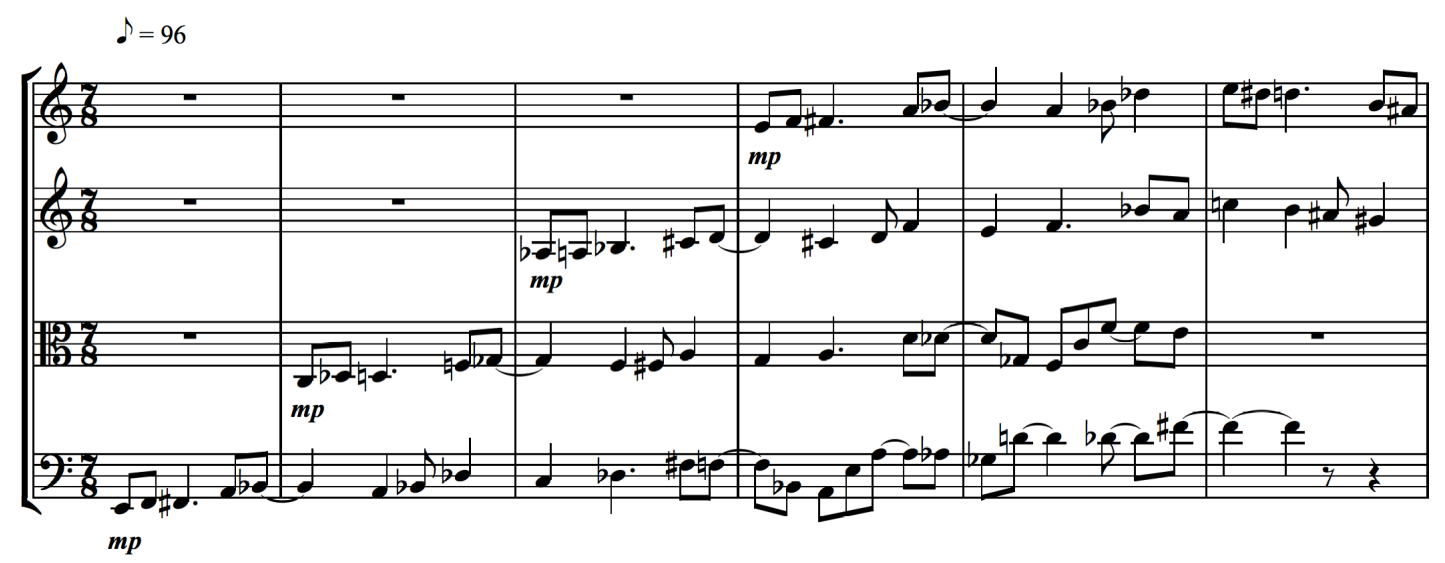

Example 4, Howard Skempton, Tendrils, measures 1-6

Harrison replies that the use of the minor sixth as the interval of transposition for each canonic entry has a useful compositional consequence too: 'minor sixths return to their original pitch two octaves higher (i.e. E-C-A flat-E again) so that bar 4 in the violin is the same as bar 1 [in the] cello'; in other words the fourth entry of each canonic figure is two octaves above the initial entry, giving a sense of completion which, in turn, necessitates a response, the next canonic entry. Each new canonic entry is both a continuation of the formal 
process out of which the entire work grows and a response to the particular conditions at each point in the unfolding music. As Harrison observes, this 'balance between process and intuition' is what makes the music 'really fascinating-both in terms of the material that disguises the joins but also the way in which [...] each canon is composed in light of the last one'. (HarrisonSkempton, 5 April 2011 10:25).

Tendrils is a remarkable achievement, its sustained and tonally ambiguous contrapuntal unfolding unlike anything else in Skempton's output. It is also like little else in the string quartet repertoire. It may sound as if it owes something to the remorseless, stripped-down counterpoint of lateShostakovitch, but the resemblance is superficial; Tendrils keeps going not because Skempton is terrified of falling silent but because he is fascinated to discover what he might think of next. As the Skempton-Harrison email exchange makes clear, the musical material is simple-phrases go up and then they come down again, with eighth-note movement predominant-but also more or less infinitely variable. There is no intrinsic reason for the music to stop when it does, except that Skempton probably felt that after 20 minutes we might have had enough. Longer notes, particularly pedal notes on $C$ in the viola and cello, appear more frequently in the latter stages of the piece, subtly preparing us for the end, but when it does come it is an agreeable surprise.

After its initial performances by the Smith Quartet, Tendrils was taken up by the Bozzini Quartet who recorded it in 2007 as part of their Canons + Hoquets CD (CQB 0704) alongside works by Jo Kondo and an earlier Skempton work for the Smith Quartet, Catch (2001). The Bozzini Quartet is based in Montreal and has a very distinctive repertoire which, although it overlaps with the other quartets considered here, also embraces a number of areas of musical activity largely ignored by other quartets, particularly those which might be loosely characterised as 'experimental', involving improvisation, different tuning systems and open, graphic or text scores. Their performances and recordings of, for example, the music of James Tenney have in turn inspired younger generations of composers to create new pieces for them which extend and develop this work. 
One such composer is Richard Glover (b.1981) whose Seventh Inversions was written for the Bozzinis and premiered by them in 2011. The entire score is shown below (example 5) and its compact layout clearly relates to many of Tenney's

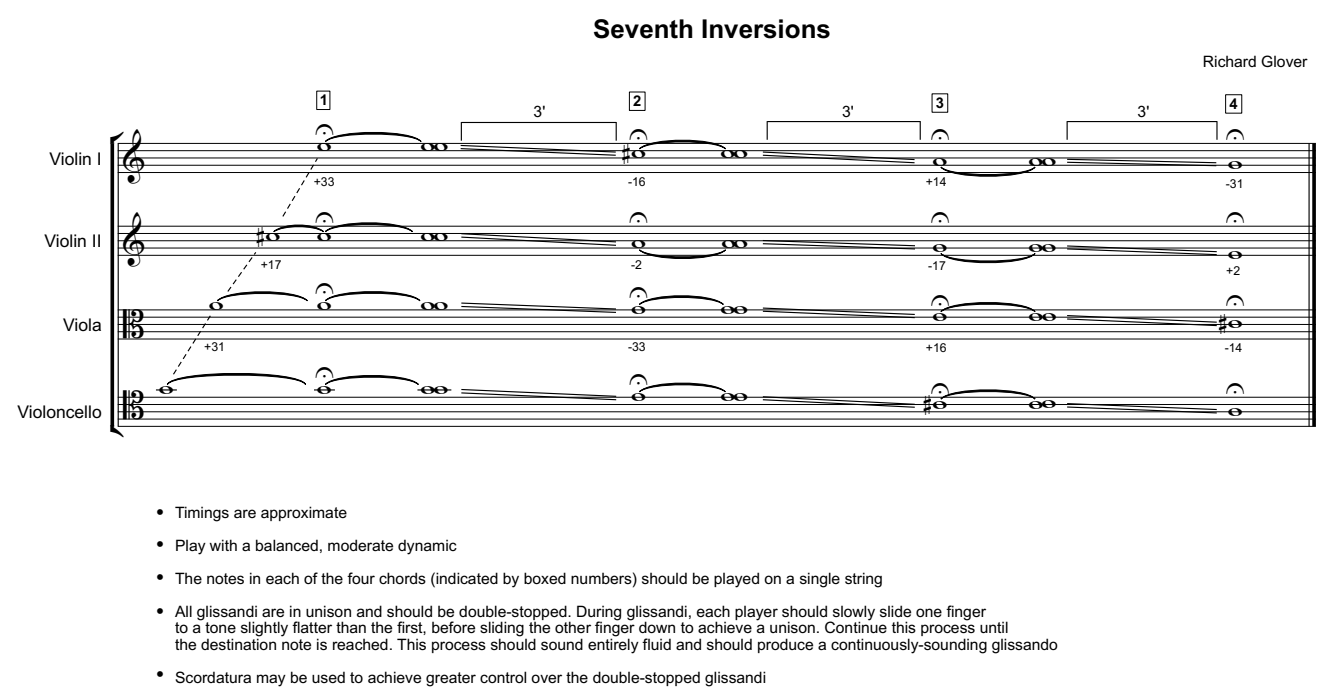

Example 5, Richard Glover, Seventh Inversions

scores, in particular to the set of ten Postal Pieces (1971) in which Tenney set himself the challenge of presenting all the material needed to perform a piece on a single postcard-sized page. As well as this visual similarity, Glover's Seventh Inversion has strong musical connections with Tenney and perhaps especially with Koan and Cellogram, the fourth and ninth of the Postal Pieces, in which solo string instruments make a series of slow glissandi.

Glover himself has said that these were 'not pieces I had in mind when I came up with the idea originally'; he says instead that 'I knew I wanted to work with gradual change, and knowing how well the Bozzini can control slow glissandi, this gave me the go-ahead'. Two elements give Seventh Inversions an individual identity which clearly distinguishes it from Tenney. The first is its tonal ambiguity; below each pitch in the upper string parts is a number giving the cent deviation from equal temperament. If these cent deviations are observed then the ratios between the pitches of the vertical aggregates at 1, 2, 3 and 4 always conform to the overtone series on $A$, with $G$ as the 
distinctively flat seventh overtone. On the other hand, the cello part contains no cent deviations, its four descending pitches making up an equallytempered dominant seventh ending on the fundamental of the chord. Glover explains this ambiguity thus: 'putting each of the four chords in their own just intonation, relating to the cello, [...] means that each chord has its own harmonic character, albeit a very nuanced one, and one that is never 'resolved' (in the purest, yet least-scientific meaning...). By keeping the allimportant cello 'out' of the JI [just intonation] tuning, it means the other instruments are acting around it throughout, and emphasis the alignment of the last chord.'

The second distinctive feature of Glover's Seventh Inversions is the rigour of its process: this is a work which does only one thing, a slow descent, and by so doing allows us as listeners to concentrate our attention on the sound of four players making staggered downward glissandi. For Glover, the issues at the heart of the work are 'process, and particularly a closure to that process. Being concerned about setting something up that could only end in one way.' It is a piece whose material determines its structure: because the material consists of the possible inversions of a four-note chord and the piece begins its descent from the third inversion of that chord, it must inevitably conclude once the root position has been reached. But, as Glover points out, the 'glissandi between each chord [each taking about three minutes to complete] mean that you don't hear the four chords as a clear sequence'. It is this balance between the music's inexorable and audible process and the unpredictability of its realisation in any one performance which makes Seventh Inversions such a fascinating experience.

The Bozzinis' enthusiasm for works which approach the string quartet at a radical tangent also inspired my chambre privée, the music which I began to plan as soon as I had accepted Richard Toop's invitation to write this article. As I suggested earlier, the string quartet is both a medium and a form and so any musician confronted by two violins, a viola and a cello is also confronted by the history of this ensemble and of the music which it has played. I decided, however, that it would be interesting to imagine a world in which these instruments had never before been put together and to write music in 
which they could be heard tentatively trying out ways of relating to one another.

chambre privée is divided into four sections, followed by a coda, and in each of the first four sections the music is made up of a series of sixteen collections of notes heard successively from 1 to 16 and then in retrograde back to 1 . All four instruments play throughout, but rather than tuning collectively they tune individually, using a limited repertoire of intervals from the overtone series which consists of all the possible combinations of overtones 5, 6, 7 and 8: the minor sixth (ratio 5:8), small fifth (5:7), perfect fourth (6:8), minor third (5:6), small third (6:7) and large second (7:8). Thus in the very first collection (see example 6), the second violin has an open string (D; each of the subsequent sections begins with a different open string on a different instrument) to which the first violin tunes (6:5, a minor third higher); the cello tunes to the first violin (5:7 plus an octave) and the viola to the cello (7:6).

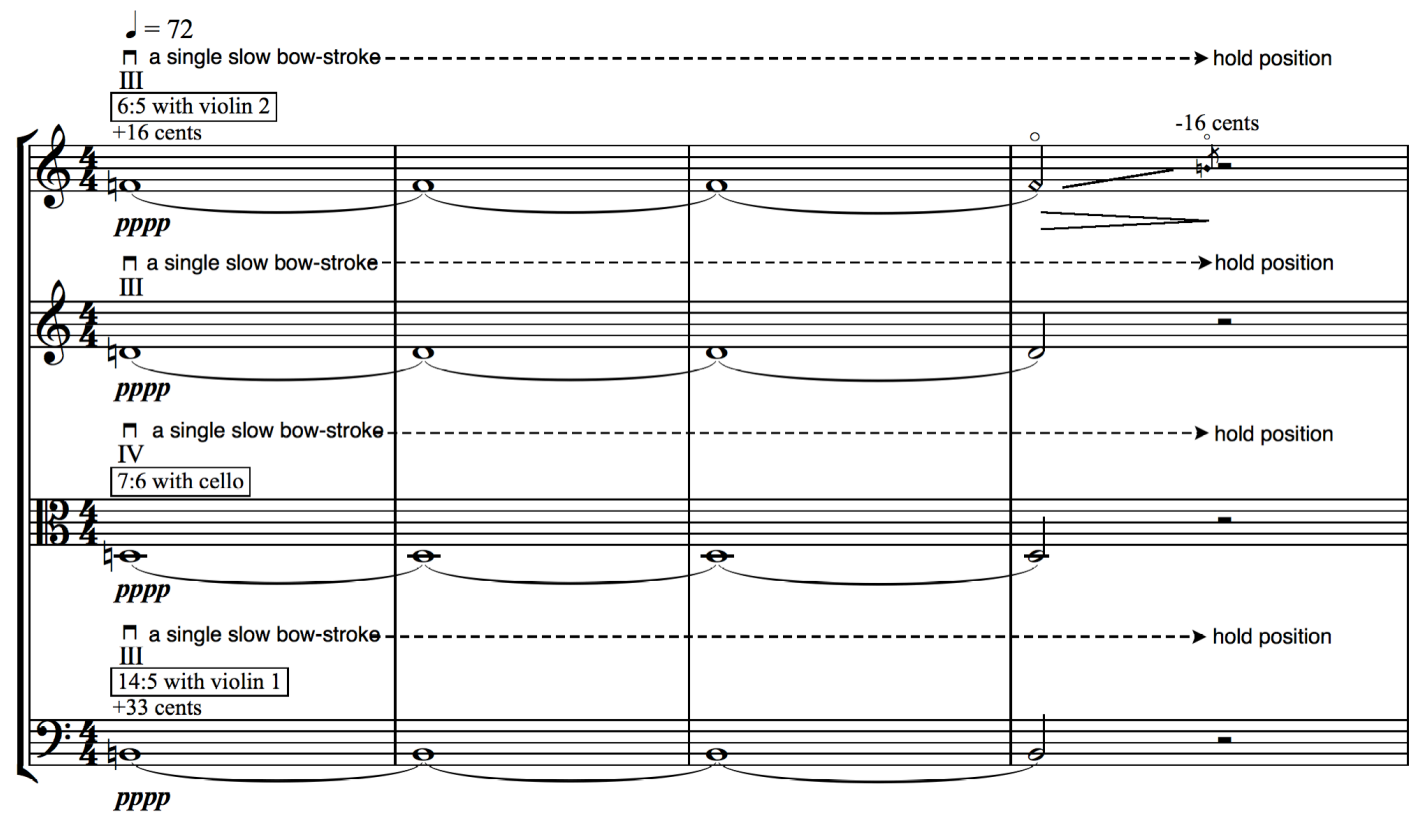

Example 6, Christopher Fox, chambre privée, measures 1-4

This process of note-to-note derivation produces very peculiar harmonies, such as the two different thirds (5:6 and 6:7) in this first collection (although the initial dynamic levels are so low that they are barely audible). But because the range of possible intervals is quite limited the music has a reassuring consistency and is a potentially fruitful refinement of the 'Harmoniad' 
principle I described ten years ago in the 'Microtones and Microtonalities' issue of Contemporary Music Review. In the works using that principle I notated pitch with a mixture of quarter- and sixth-tone accidentals to indicate acceptable approximations of the pitches generated by the harmoniad process. In chambre privée, however, I decided to notate the music by combining the semitonal accidentals from standard equal temperament with numerical indications of cent deviations, a method I had previously used in the brass trio Hidden Consequences (2009). It is a method which not only acknowledges the absence of a consistent division of the octave but also enables very precise tuning, particularly when, as the Bozzinis do, the players use pitch trackers to check the accuracy of their intonation.

As chambre privée evolves it gradually gets a little faster, as if the players were becoming more confident in what they are doing, and towards the middle of each of the first three sections their entries become progressively less coordinated, again suggesting a growing confidence in their ability to act independently and yet remain in consort. In the fourth section the instruments almost immediately start to play independently and then start sliding around, perhaps enjoying the freedom they seem to have achieved, or perhaps in an ever more desperate attempt to regain the system of organisation with which the music began.

Whatever the significance of these continuous glissandi, some sort of crisis is triggered and the music breaks down. The second violin, viola and cello form a trio and play a slow sequence of harmonies, the violinist and violist resting their instruments on their knees and playing them as if they were viols. Meanwhile, the first violinist retunes his instrument to a scordatura based on the overtone intervals on which all the harmonies in the work have been based: the fourth string $G$ remains, but the third string is tuned down to make a 3:4 C above the $\mathrm{G}$; the second string becomes a low $\mathrm{G}$ flat, 5:7 above the $\mathrm{C}$, and the top string comes down to E flat, the inverted 5:6 above the second string. In this new tuning he plays a cadenza whose figuration is in part derived from some of the virtuoso writing in Biber's 'Mystery' Sonatas. 
It might be argued that, having set out to write music about how a string quartet might work as an ensemble if its participants knew nothing of its history, I eventually created a work whose conclusion-the first violin goes off on his own and the remaining trio discover that they can manage quite well without him-suggests that the ensemble doesn't work, brought down by the treble imbalance I mentioned at the beginning of this article. It might also be argued that, in attempting to imagine a string quartet without a history, I created a work which makes very knowing references both to the pre-history of the quartet in earlier forms of string solo and consort music and to the primus inter pares status of the first violinist, often a source of friction within quartets.

Whatever the conclusion listeners may draw, my own response to the experience of writing chambre privée and, at the same time, writing this article is that the string quartet remains one of the most fascinating groupings within the classical music instrumentarium. Its revitalisation, first by the Arditti and Kronos Quartets and subsequently by the ensembles who have followed their example, has created perhaps the most interesting and representative collection of new works to have emerged in the last 40 years. Just as in the era of Haydn, Mozart and Beethoven, composers today write their most innovative, personal and serious music for the string quartet, knowing that there are dedicated ensembles with the technical and imaginative capacity to play anything they choose to write.

\section{REFERENCES}

Amis, K. (1954). Lucky Jim, 47-56. London: Gollancz.

Clarke, J. (2003). String Quartet. London: G. Ricordi \& Co.

Fallas, J. (2012). 'Conditions of immediacy: Howard Skempton in interview', Tempo, Vol.66, No.262, October 2012, 13-28.

Finnissy, M. (2007). Second String Quartet. Oxford: Oxford University Press.

Finnissy, M. (2009). Third String Quartet. Oxford: Oxford University Press.

Finnissy, M. and Fox, C. (2011). Private email exchange.

Fox, C. (2003). Contemporary Music Review

Glover, R. (25 February 2013). Private email to Christopher Fox. 
Harrison, B., and Skempton, H. (4-5 April 2011). Private email exchange. Harvey, J. (2003). String Quartet No.4. London: Faber Music.

Harvey, J. (2004). 'The Genesis of Quartet No.4', in Identity and Difference: Essays on Music, Language and Time, Leuven: Leuven University Press Skempton, H. (2004) Tendrils [score]. Oxford: Oxford University Press. 